\title{
Philosophiques
}

\section{À qui le droit de taxer ? Être membre d'un État et les enjeux fiscaux qui en découlent}

\section{Allison Christians et Nicolas Benoît-Guay}

Volume 43, numéro 1, printemps 2016

URI : https://id.erudit.org/iderudit/1036472ar

DOI : https://doi.org/10.7202/1036472ar

Aller au sommaire du numéro

Éditeur(s)

Société de philosophie du Québec

ISSN

0316-2923 (imprimé)

1492-1391 (numérique)

Découvrir la revue

Citer ce document

Christians, A. \& Benoît-Guay, N. (2016). À qui le droit de taxer ? Être membre d'un État et les enjeux fiscaux qui en découlent. Philosophiques, 43(1), 127-132. https://doi.org/10.7202/1036472ar d'utilisation que vous pouvez consulter en ligne. 


\title{
À qui le droit de taxer? Être membre d'un État et les enjeux fiscaux qui en découlent
}

\author{
ALLISON CHRISTIANS \\ NICOLAS BENOÎT-GUAY*
}

\section{Introduction}

Dans son livre Catching Capital: The Ethics of Tax Competition, Peter Dietsch brosse un portrait à la fois pertinent et préoccupant des principaux enjeux liés au droit fiscal international ${ }^{1}$. Là où trop souvent les spécialistes du droit fiscal pourraient s'égarer dans la discussion de technicités, Dietsch propose une approche nouvelle du problème, qui considère tant le droit que l'économie et la philosophie. En ce sens, son livre représente un ajout majeur dans un domaine où l'interdisciplinarité est tout aussi nécessaire que trop souvent absente.

Le thème central du livre est celui de la compétition fiscale qui oppose les États à l'échelle internationale. Selon Dietsch, dans le cadre juridique actuel, cette compétition a des effets nocifs, car elle limite la "souveraineté » des États en restreignant les choix de politiques fiscales qui s'offrent à eux. La souveraineté fiscale implique que ceux-ci soient autonomes dans la réalisation de leurs choix fiscaux. Cependant, en devenant acteurs d'une économie globalisée, les États cèdent systématiquement cette autonomie, ce qui suggère que la souveraineté fiscale n'est pas l'enjeu principal de la compétition fiscale. Aux fins de cette contribution, nous assumerons donc que la compétition fiscale entrave l'exercice souverain des États. En effet, ceux-ci sont contraints de modifier leur politique fiscale pour essayer de garder leur économie compétitive. Si un État n'opère pas les ajustements requis, il risque de perdre les revenus qu'il aurait pu tirer, par exemple, d'une croissance économique alors réduite par une diminution des investissements. Catching Capital: The Ethics of Tax Competition est une tentative de réponse au problème de la compétition fiscale qui, bien qu'elle demeure imparfaite, a le mérite de présenter une solution détaillée et structurée.

\section{Le principe d'appartenance}

Dans cet article, nous nous concentrerons sur un aspect précis de la proposition de Dietsch, soit le "principe d'appartenance». Le principe d'appartenance établit que toute personne peut être assujettie à l'impôt d'un État si elle en est membre. La logique découlant de ce principe est qu'un État ne

* Allison Christians, H. Heward Stikeman, Chair in Taxation, McGill University Faculty of Law; Nicolas Benoît-Guay, B.C.L./LL.B., McGill University 20I5; LL.M. McGill University, expected 2016.

1. Peter Dietsch, Catching Capital: The Ethics of Tax Competition, OUP USA, 20 I 5. 
peut assujettir une personne qui n'en est pas membre. Pour la personne, cela veut dire que seul l'État dont elle est membre peut la contraindre à payer l'impôt. Néanmoins, ce principe ne se limite pas à établir une relation entre un État et ses contribuables; il a aussi pour but de régir les conflits entre États. Si une personne est membre de plus d'un État, alors chacun aura le droit de la taxer, d'où résultera le problème de la double taxation ${ }^{2}$, alors que l'État dont cette personne n'est pas membre ne pourra le faire. De façon générale, le principe d'appartenance vise donc à catégoriser les contribuables en les attribuant aux États dont ils sont membres.

Quoique semblant aller de soi, le principe d'appartenance pourrait avoir de grandes répercussions en droit fiscal international, tout dépendant de la définition donnée à la qualité de «membre ». Selon Dietsch, est membre d'un État toute personne, physique ou morale, qui «bénéficie des services publics et des infrastructures» fournis par celui-ci. Cependant, il soutient que cette définition de l'appartenance est conforme aux concepts déjà existants en droit fiscal international de résidence et de source.

Le principe de résidence établit qu'un État peut taxer toute personne qui est juridiquement identifiée comme résidente, tandis que le principe de source reconnaît comme contribuable toute personne générant un revenu à l'intérieur de cet État. Bien que séduisant théoriquement, étant donné leur catégorisation claire du revenu et du contribuable, chacun des principes devient difficilement applicable dans les faits. La complexité pratique des notions de revenu et de contribuable empêche en effet une application claire de ces principes, ce qui permet aux États de modeler leur application à leur avantage. Les carences systémiques liées à l'application de ces principes ont amené quelques juristes à rejeter en théorie et en pratique les principes de résidence et de source comme fondements normatifs du droit fiscal international. En proposant le principe d'appartenance, Dietsch semble vouloir remédier à ce vide, d'où la nécessité d'en évaluer la valeur normative.

La définition du principe d'appartenance choisie par Dietsch soulève deux séries de questions. La première découle du fait que, selon lui, le principe d'appartenance s'applique tout autant aux personnes physiques qu'aux personnes morales. De prime abord, ce traitement égal de la personne physique et de la personne morale est justifié, car chacune est reconnue comme titulaire de droit au sens de la loi. Cependant, l'impôt ne s'applique pas également à la personne morale et à la personne physique, qui ont chacune leur propre régime fiscal, ce qui pourrait suggérer un traitement distinct au regard du principe d'appartenance. Une autre difficulté, plus importante, provient du don d'ubiquité inhérent à la personne morale. Les conséquences

2. Il y a double taxation lorsqu'un revenu est taxé deux fois. Empêcher la double taxation du revenu a longtemps été l'un des objectifs principaux du droit fiscal international, car on considérait que la double taxation résulterait en une surtaxation du revenu ce qui agirait comme un désincitatif au commerce international. 
découlant de cette capacité à se multiplier sur le plan fiscal posent problème à l'application du principe d'appartenance, car la personne morale peut appartenir à de très nombreux États, et cela à des degrés multiples. Finalement, le principe d'appartenance devrait peut-être aussi tenir compte du fait qu'une personne morale, en tant qu'entité juridique désincarnée, n’a pas de sentiment d'appartenance à une communauté. Ce sont plutôt ses dirigeants, ses employés et ses clients qui développent un attachement à leur communauté. Or chacun d'eux peut se trouver dans des États différents. Cette distinction est importante, car plusieurs études suggèrent aujourd'hui que l'acte de payer ses impôts est en partie expliqué par le sentiment d'appartenance du contribuable à une communauté 3 .

\section{L'appartenance comme découlant du bénéfice}

Bien qu'il y ait beaucoup à dire sur le dernier enjeu soulevé, notre attention portera maintenant sur un autre aspect du principe d'appartenance, soit l'idée qu'un membre est celui qui bénéficie des services publics et des infrastructures fournis par l'État. La définition d'appartenance choisie par Dietsch sous-entend qu'être membre d'une communauté implique nécessairement le bénéfice qui peut en être tiré. En droit fiscal international, la théorie du bénéfice soutient qu'une personne a une obligation fiscale à l'intérieur d'un État à partir du moment où elle bénéficie de ses services publics. Pour sa part, Dietsch distingue services publics et infrastructures, bien qu'il n'explique pas cette distinction ni le rôle qu'elle joue dans la compréhension du principe d'appartenance. Pour la contribuable, les services publics peuvent être considérés comme une diminution des coûts d'opération: par exemple, l'existence de routes et de ponts est essentielle au déplacement de biens matériels. Les États jouent alors un rôle indispensable en permettant le commerce à une échelle internationale. L'impôt est en conséquence la contrepartie aux services et biens fournis par l'État. Quoiqu'établissant ainsi le droit de taxer de celui-ci, l'auteur n'en déduit pas que le taux d'imposition doit être proportionnel aux services rendus, rejetant de ce fait ce second aspect de la théorie du bénéfice.

Afin de décider qui est membre d'un État, il devient nécessaire de pouvoir déterminer si une personne bénéficie ou non des services publics (et, selon Dietsch, des infrastructures) fournis par celui-ci. Or ce point n'est pas développé par l'auteur, qui ne définit pas les concepts de services publics et d'infrastructures, pas plus qu'il ne délimite la portée de l'idée de bénéfice à l'intérieur du principe d'appartenance. Cette omission est problématique, car il devient alors difficile d'appliquer le principe d'appartenance avec clarté.

3. À ce sujet, voir Erzo F. P. Luttmer et Monica Singhal, "Tax Morale» (20I4), 28, 4, J. Econ Perspect, I49-I 68 qui fait la revue des principales études sur cet enjeu. 
Bien qu'il ne précise pas sa compréhension du concept de bénéfice, Dietsch conclut que le principe d'appartenance éliminerait deux formes de compétition fiscale jugées nocives, soit la compétition pour les placements de portefeuille et la compétition pour les profits théoriques créés par les pratiques de transfert de prix. Suivant le raisonnement de Dietsch, ces deux formes de compétition fiscale seraient éliminées, car les personnes morales qui se prévalent, à des fins d'évitement fiscal, du système d'impôt des pays où les niveaux d'imposition sont bas ou même inexistants ne seraient alors pas considérées comme membres de ces pays. De ce fait, le principe d'appartenance établit qu'une contribuable ne peut prétendre avoir une obligation fiscale envers un État dont elle n'est pas membre et, corollairement, que celui-ci ne peut revendiquer de juridiction sur une part du revenu d'un nonmembre. La conséquence pratique serait alors que le revenu des entreprises profitant de la compétition pour les placements de portefeuille et de la compétition pour les profits théoriques serait imposé par les pays résidents ou sources qui, eux, pourraient prétendre à l'appartenance de la contribuable.

L'application que fait Dietsch du principe d'appartenance sous-entend du moins une conception plus restreinte de la théorie des bénéfices. Il s'ensuit alors qu'il devient possible d'identifier ce que l'auteur entend par services publics et infrastructures. Dans cette approche restreinte, les services publics et les infrastructures se limitent à tout exercice du gouvernement qui est directement lié à la génération du profit de la contribuable. Cela peut passer par la formation de la main-d'œuvre, les subventions, l'existence d'un système d'éducation et de santé, etc. Corrélativement, un pays ne participe pas directement à la génération du profit de la contribuable quand il n'est pas impliqué en fournissant ou en subventionnant directement ou indirectement le capital productif, qu'il s'agisse de l'équipement et de l'outillage, de l'acquisition de matières premières ou encore de la main-d'œuvre.

Cependant, il est possible d'adopter une approche de la théorie du bénéfice qui inclut d'autres formes de bénéfices. Principalement, suivant la nomenclature de Dietsch, il faut ici considérer le système légal de chaque pays en tant qu'infrastructure. En effet, un pays qui est capable de garantir la validité des contrats, de protéger les droits de propriété intellectuelle ou de faciliter le financement fait bénéficier la contribuable. De plus, il est même possible d'adopter une approche de la théorie du bénéfice encore plus large, car l'activité économique d'une multinationale se déploie dans un espace économique globalisé. En fait, cet espace dont bénéficie la multinationale résulte d'un long processus de coopération impliquant les pays à une échelle mondiale. Ce processus a été possible grâce à l'inter-connectivité des langues et des cultures qui a favorisé les déplacements de capitaux, le commerce de biens et de services, les contrats multipartis nécessaires à la construction de chaînes de production complexes.

Le bénéfice découlant de cet espace économique globalisé pour l'entreprise ne peut être attribué à un ou plusieurs États, car il correspond à la 
valeur ajoutée résultant d'un exercice de coopération impliquant tous les États. Suivant cette définition plus large du bénéfice, le principe d'appartenance ne règlerait donc pas les problèmes liés à la compétition pour les placements en portefeuille et pour les profits théoriques, car les États que l'on associe généralement aux paradis fiscaux offrent d'une part l'accès à des infrastructures légales, et d'autre part participent à la libéralisation des échanges.

La conclusion de Dietsch soutenant que ces deux formes de compétition seraient éliminées par le principe d'appartenance suggère une application restreinte de la théorie du bénéfice. Cependant, sa proposition d'adopter un système de la «répartition globale selon une formule préétablie » suggère plutôt une compréhension large de l'idée de bénéfice. Selon cette formule, la charge fiscale d'une entreprise est mesurée en évaluant ses ventes, ses valeurs mobilières ainsi que les salaires payés à ses employés. En outre, un pays qui ne serait ni résidence ni source pourrait prétendre avoir le droit d'imposer une part du revenu de la contribuable si les produits ou services de celle-ci y sont vendus. Suivant l'approche par les bénéfices, cette formule est justifiée, car la vente des produits n'est possible qu'à partir du moment où, par exemple, les contrats de vente sont garantis. Or l'adoption d'un système de la répartition selon une formule préétablie n'élimine pas les risques liés à l'évitement fiscal. Par exemple, la contribuable pourra localiser stratégiquement le lieu de la première vente, plutôt que de localiser stratégiquement le lieu du profit. De ce fait, le principe d'appartenance doit pouvoir expliquer pourquoi une multinationale serait considérée comme bénéficiaire seulement dans le pays de la vente finale et non dans les pays servant de subsidiaires.

Le principe d'appartenance tel que développé par Dietsch semble donc incohérent, car il s'appuie d'une part sur une conception restreinte du bénéfice quand vient le temps de discuter l'enjeu des paradis fiscaux, et d'autre part sur une approche plus large quand il préconise l'adoption d'une répartition selon une formule préétablie où les ventes sont prises en compte. Ce décalage entre les deux approches est problématique, car il illustre bien que le principe d'appartenance n'est pas neutre et qu'il peut ainsi servir les intérêts de certains pays. Conséquemment, il n'est pas évident qu'il y aurait consensus sur son application, car les intérêts des pays divergeraient quant à la conception appropriée du bénéfice.

L'appartenance comme forme de bénéfice semble donc mériter une étude plus approfondie, car, sans précision, le principe d'appartenance peut se révéler caduc. Bien que ce commentaire n'ait pas la prétention de régler cet enjeu, il semble que l'approche large soit à privilégier. En effet, les systèmes de droit jouent aujourd'hui un rôle important dans l'économie mondiale en réduisant grandement le risque associé aux transactions du commerce international par les contrats d'assurances, le réseau bancaire, le système d'échange des monnaies, etc. Dans ce contexte, il devient difficile de 
défendre que les multinationales ne bénéficient pas des services publics et des infrastructures que l'on retrouve dans les paradis fiscaux, et cela bien au-delà du faible niveau d'imposition. 